\title{
Circulating tumor cells as a new and additional approach to follow-up patients with serous low-grade ovarian adenocarcinoma - a case report and review of the literature
}

\author{
Marcelo Corassa ${ }^{1,2}$, Andrea Paiva Gadelha Guimarães', Solange Moraes Sanches', Marcello Ferretti Fanelli' \\ Bruna Maria M. Rocha², Alexandre Andre Balieiro A. da Costa ${ }^{1}$, Vanessa Alves ${ }^{2}$, Glauco Baiocchi ${ }^{3}$ \\ and Ludmilla T. Domingos Chinen ${ }^{2 *}$
}

\begin{abstract}
Background: Current methods for follow-up of Ovarian Cancer (OC) are widespread, especially with CA125, which, however, is not a perfect biomarker and thus further investigation for new methods of evaluation are warranted. The feasibility of Circulating Tumor Cells (CTCS) in advanced OC was investigated in this case report.

Case presentation: A 19-year-old woman with advanced low-grade serous papillary adenocarcinoma and relapsed disease did not have a CA125 correspondent with disease relapse. CTCs were evaluated, compared with CA125 and with image exams. Relapses were not correspondent to elevations of CA125. CTCs demonstrated usefulness, being proportional to major disease relapse, especially in the peritoneum. CTCs may be used as a complementary diagnosis tool when marginal/small increases in CA-125 levels are observed.

Conclusions: In OC, CTCS can be an important tool to predict recurrence, response to treatment and improve the quality of decision-making, in order to offer the best treatment to a determined group of patients.
\end{abstract}

Keywords: Ovarian cancer, CA125, Circulating tumor cells, ISET, Low-grade ovarian adenocarcinoma, Follow-up, Young patient

\section{Background}

Epithelial ovarian cancer is the most lethal cancer of the female genital tract and has a significant incidence in both developed and undeveloped countries. It is predominant in perimenopausal and postmenopausal period with up to $90 \%$ of the cases occurring after the age of 40 years [1]. Before this age, tumors of stromal and germinative lineage account for the majority of cases, and the clinical behavior is considerably different [2].

\footnotetext{
* Correspondence: Itdchinen@gmail.com; ludmilla.chinen@cipe.accamargo.org.br

${ }^{2}$ International Research Center, A.C.Camargo Cancer Center, Rua Taguá, 440. Liberdade, São Paulo, SP 01508-010, Brazil

Full list of author information is available at the end of the article
}

The clear majority of data regarding epithelial ovarian cancer therapy arise from trials comprising populations of women who lived past the fifth decade, and mainly with high-grade adenocarcinomas. Low-grade tumors are characterized by a different clinical presentation and behavior: younger patients, relative chemoresistance and prolonged overall survival [3, 4]. Moreover, low-grade tumors are more difficult to follow-up, since CA-125 levels are normally not as high as in high-grade tumors, demanding alternative approaches to identify recurrence and relapse [5-7].

In this report, we present a case of a 19-year-old patient who was diagnosed with a low-grade adenocarcinoma of the ovary and treated according to standard protocols. The patient was evaluated for CTCs after a 
first recurrence and followed up with periodic dosages upon clinical decision.

\section{Case presentation}

\section{Clinical evaluation}

A Caucasian woman, initially 19 years-old, had a story of persistent abdominal cramps. Late in 2012 an abdominopelvic ultrasound showed a heterogeneous nodular expansive mass occupying the vesicouterin space, with $5,0 \times$ $3,2 \mathrm{~cm}$ in the largest dimension. In December 2012, an abdominopelvic magnetic resonance (MRI) demonstrated similar findings, and additionally peritoneal implants without lymph node enlargement. No primary tumor was suggested after the MRI. The primary diagnostic hypothesis was an immature teratoma of the ovary.

\section{Initial treatment}

The patient underwent biochemical investigation, clinical and radiological staging. The biomarkers were characteristic of an epithelial tumor as it follows: CA-125 278.8 UI/ $\mathrm{mL}$ (Reference Value - RV $<35 \mathrm{UI} / \mathrm{mL}$ ), Carcinoembriogenic Antigen $(C E A)<0.5 \mathrm{ng} / \mathrm{mL}(\mathrm{RV}<5 \mathrm{ng} / \mathrm{mL})$, CA19$913.7 \mathrm{UI} / \mathrm{mL}(\mathrm{RV}<39 \mathrm{UI} / \mathrm{mL})$, hCG (human chorionic gonadotrofin $)<0.5 \mathrm{nUI} / \mathrm{mL}(\mathrm{RV}<2 \mathrm{nUI} / \mathrm{mL})$, alpha fetoprotein $(\mathrm{AFP})<0.6 \mathrm{ng} / \mathrm{mL}(\mathrm{RV}<13.7 \mathrm{ng} / \mathrm{ML})$.

In January 2013, an exploratory laparotomy was performed, with biopsies of right ovary, sigmoid implant, omentum and left uterine tube. Every biopsy demonstrated a low-grade serous papillary adenocarcinoma of the ovary and peritoneal cytology was positive for malignant cells. In February 2013, a complete cytorreduction was performed with pelvic and retroperitoneal lymphadenectomy. The histopathologic analysis of the right ovary reported a lowgrade serous papillary cystadenocarcinoma, with invasion of right uterine tube, uterine corpus, adjacent colon, cecal appendix, omentum, and multiple peritoneal areas. Six of 67 lymph nodes were positive for malignancy. There were areas containing cells with high-grade nuclear index and low mitotic activity, which suggests the presence of scattered high-grade neoplastic areas.

She was admitted to the Medical Oncology Department in March 2013. The therapeutic plan consisted of 6 cycles of adjuvant Carboplatin with a predicted area under the curve (AUC) of 5 with paclitaxel $175 \mathrm{mg} / \mathrm{m}^{2}$ every 21 days. The patient was offered for the dosage of circulating tumor cells (CTC) with complete acceptance. Chemotherapy cycles were completed in August 2013, without any delays or grade 3 or 4 toxicities. Follow-up was started in September 2013. No previously identified pathogenic mutations in BRCA1 and BRCA2 genes were found.

\section{Disease recurrence}

Planned follow-up was provided with imaging studies every three months, combined with laboratory tests and
CA125 dosage. CTC dosage was paralleled to ambulatory visits and not deemed as part of the follow-up protocol. The images did not show progressive disease (PD) until April/2014, when the CA125 modestly rose (14.3 UI/mL in December 2013 to $34.2 \mathrm{UI} / \mathrm{mL}$ in April/ 2014 and continued to rise until July/2014, with $47.2 \mathrm{UI} /$ $\mathrm{mL}$ peak). An abdominopelvic $\mathrm{CT}$ was performed, showing disease recurrence.

In August/2014 an optimal secondary cytorreduction was performed. The pathological report showed recurrence of the disease: low grade serous papillary cystadenocarcinoma of the ovary with infiltration of vesical peritoneum, sigmoid colon, obturatory fossa, spleen, stomach, duodenum, liver, gallbladder, and diaphragm, including other peritoneal areas.

After the secondary cytorreduction, a new course of chemotherapy was initiated with Carboplatin AUC 4 day $1+$ Gemcitabine $1000 \mathrm{mg} / \mathrm{m}^{2}$ days 1 and $8+$ Bevacizumab $15 \mathrm{mg} / \mathrm{kg}$ [8] in September/2014. She underwent a difficult course of chemotherapy for 6 cycles, requiring granulocyte colony stimulating factor (G-CSF) support. Maintenance bevacizumab was offered until January/ 2016, when PD was identified in a nodule in abdominal wall. Third line chemotherapy was initiated with Carboplatin AUC 5 day $1+$ Pegylated Liposomal Doxorubicin $30 \mathrm{mg} / \mathrm{m}^{2}$ every 28 days.

\section{Methods}

This patient was evaluated according to best practice protocols for Ovarian Cancer. Every treatment was conducted at A.C.Camargo Cancer Center, following every rule for patient safety.

\section{Isolation, counting and characterization of circulating tumor cells}

Blood samples were collected from patient after she had signed the informed consent to study with circulating tumor cells in solid tumors, which has been approved by the Local Research Ethics Committee of A.C. Camargo Cancer Hospital, São Paulo, Brazil (protocol number: 1367-10).

Blood $(8 \mathrm{~mL})$ was collected in EDTA tubes, stored at room temperature under homogeneization for up to $4 \mathrm{~h}$, and treated by ISET (Isolation by Size of Tumor cells (ISET) (Rarecells Diagnostics, Paris, France) according to manufacture procedure. This methodology uses polycarbonate membrane with $8-\mu \mathrm{m}$-diameter cylindrical pores to isolate intact CTCs from blood through direct filtration, based on the concept that of the larger size of tumor cells as compared with leukocytes.

Rapidly, $8 \mathrm{ml}$ blood was diluted on ISET buffer, transferred to the ISET block and filtered. Cells that have more than $8 \mu \mathrm{m}$ were maintained by negative pressure in the membrane, that was stored at $-20{ }^{\circ} \mathrm{C}$ until time of analysis. 
Cells were considered as CTCs if they have presence of hypercromatic nucleus, irregular shape, high cytoplasm nucleus ratio ( $>0.8$ ), stain negatively for CD45 and have nuclear size $\geq 12 \mu \mathrm{m}$ (Krebs et al., 2012). For cell counting, ISET membrane spots were cut out and used for single immunocytochemistry (DAB+/Dako $\left.{ }^{\mathrm{TM}}\right)$ for exclusion of leucocytes (CD45; 1:100; clone 2B11+ PD7/26, Dako ${ }^{\mathrm{TM}}$ ), according to the manufacturer's instructions. Eight spots on the ISET filter were used for counting, corresponding to $8 \mathrm{~mL}$ of blood $[9,10]$.

\section{Circulating tumor cells analysis}

The chronologic relationship between dosages of CA125 and CTCs is shown in Fig. 1. At the first evaluation, before receiving any surgical or chemotherapeutic treatment CTC dosage was $7.5 \mathrm{CTCs} / \mathrm{mL}$. In that opportunity, the CA125 was $26.8 \mathrm{UI} / \mathrm{mL}$. After this first dosage, the patient underwent the first treatment course (Carboplatin AUC $5+$ Paclitaxel $175 \mathrm{mg} / \mathrm{m}^{2}$ ). Her following evaluations did not show any prediction of progression for CA125 until ascension in CTC dosage (see Fig. 1) with a peak of $3.6 \mathrm{CTCs} / \mathrm{mL}$ in April/2014, and an only modest ascension in CA125 (33.9 $\mathrm{UI} / \mathrm{mL}$ ). In July 2014, CTCs and CA125 were still rising (5.3 CTCs $/ \mathrm{mL}$ and $45.0 \mathrm{UI} / \mathrm{mL}$ respectively), with demonstration of PD of images in August 2014. After the end of the proposed second line chemotherapy and during bevacizumab maintenance CTCs levels were stable.

Follow-up was continued during bevacizumab maintenance, with a novel and slow rise until PD. In March/ 2016 there was an increase on CA125 without an increase in CTC. Later, on May/2016, CTC levels rised, with a stable level of CA125. This finding is considered as related to the low burden of disease on the abdominal wall and probable stability of peritoneal disease, thus, CTCs may not be of analytic value for superficial progressive disease, such as in this case, when progressive disease was detected only in the abdominal wall.

To evaluate the direct correlation between CTC and CA125 levels, a paired samples $T$-test was performed considering the value for every data-point using IBM SPSS Statistics 20.0 for Macintosh. This test showed a 0.868 significance when comparing the 12 paired dosages for both variables. The results of this test confirmed the impossibility to draw a definitive conclusion between CTC and CA125 and the possible correlation among both, considering a statistically significant $\mathrm{p}$ value of $<0.05$.

\section{Discussion}

Evaluation and measurement of burden of disease in ovarian cancer has always been a challenge. Due to its

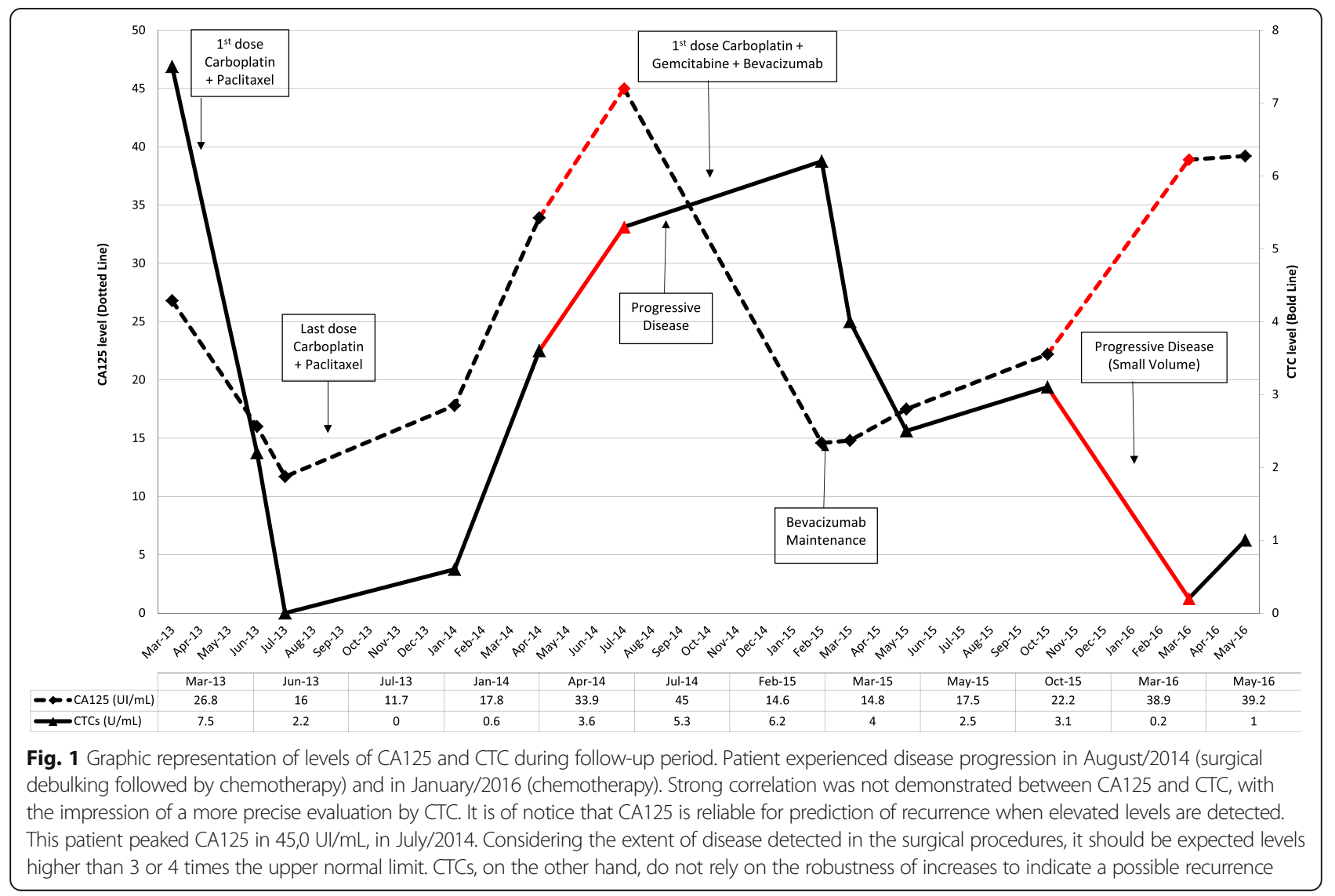


characteristics, it is difficult to identify response or recurrence in imaging studies. Historically, CA125 is the most important biomarker for follow-up in ovarian cancer [11-13]. It has a direct relationship with burden of disease, and has been used to evaluate treatment response and recurrence.

A high baseline level of CA125 is often associated with worst outcomes for primary treatment [14], even in patients with cancer of low malignant potential [15]. Similarly, a high level of CA125 often demonstrates a massive recurrence. Additionally, CA125 decrements are useful for determining effectiveness of therapy in the neoadjuvant setting and can predict optimal cytorreduction and better outcome [16].

Much is being done at improvement in the quality of CA125 as a biomarker. Nevertheless, concrete data is lacking to allow the physician to rely only on its levels to make definite decisions, since it is not able to predict response or recurrence in some patients [17]. For instance, in some ovarian cancer histological types, such as clear cell carcinoma and low-grade adenocarcinoma, frequently the CA-125 levels do not correlate with extent of disease. The levels of CA125 sufficient to warrant the suspicion for recurrence are normally robust, over 3 or 4 times the upper reference limit of the test. The ideal cutoff, however, is not defined, opening a role for CTC kinetics analysis.

CA-125 is also often expressed in patients without cancer, and can be detected in recurrences when disease is measurable. The levels of CA125 are higher with increasing age, use of hormone therapy, former smoking and history of breast cancer, but can be lower after hysterectomy, in current smokers, obese and non-white women $[18,19]$. Moreover, the capacity of determining good prognosis in early declines from baseline [20] is lost if the neoplasm doesn't express significant levels of CA125.

According to recent data, it is estimated that $95 \%$ of recurrences of ovarian cancer can be detected by using the current NCCN guidelines for follow-up [21]. This results in $5 \%$ of women that are not covered by typical tools of follow-up and should be evaluated by different methods. In this setting, the evaluation of CTCs and disseminated tumor cells (DTCs), in the bone marrow, arise as a prominent tool for follow-up of specific groups of patients [22-24].

This case describes a very young woman bearing an unusual neoplasm for her age, what makes the optimal choice of treatment and decisions regarding her follow-up challenging. The patient had a recurrence with a great extent of peritoneal disease, without pronounced elevation of CA125 or burden of symptoms. Of notice, it was expected a very important elevation in the CA125 levels, and only a modest one was encountered. The case itself and the anterior considerations encouraged the clinicians to offer her the CTC dosage protocol. A negative assay for CTCs for this patient would be precisely useful. On the other hand, the elevation of CTCs could demonstrate a strong evidence of recurrent and extensive disease [22]. Moreover, since it seems that CTCs have the ability to disseminate, a positive result points out an increased risk for extra-peritoneal disease [25], what makes the choice for systemic therapy even more important.

The majority of data regarding CTCs comes from breast, colon and prostate cancer [26]. Studies with ovarian cancer are not frequent and controversial. Marth et [25] were unable to demonstrate a positive correlation of CTC detection and prognosis, with equivalent progression free survival (PFS) and overall survival (OS) between patients with positive and negative CTCs in blood samples. To isolate CTCs they used density gradient centrifugation followed by immunomagnetic labeling with MOC31, an epitope regularly expressed on ovarian carcinoma cells. Judson et al. [27] confirmed absence of prognostic value for CTCs when dosing them by the same method, but using a pool of antibodies (anti-cytokeratin 7, 8, 18 and 20, TFS-2 and EGFR) before cytorreduction surgery. However, the sample analyzed in this study was small and the follow-up short. Other groups, working with different assays, showed different results. Fan et al. [27] suggested that patients with elevated CTCs might predict shorter disease free survival (DFS) using Vita-Assay ${ }^{\mathrm{TM}}$ (an immunolabeling method) for evaluation of CTCs. They also showed that in patients with high-grade adenocarcinoma there was a direct relationship between higher levels of CTCs, more aggressive disease, and higher levels of CA125. Poveda et al. [28] also found a direct connection between elevated CTCs and shorter PFS and OS. The study conducted by Poveda was a multicentric phase III study, enrolling 672 patients. The CTCs were investigated using the CellSearch $^{\mathrm{TM}}$ system, using anti-EPCAM antibodies, conjugation of anti-cytokeratin and decontamination of leucocytes with anti-CD45 staining. The univariate analysis found that patients with elevated CTCs had shorter PFS $(6,6 \times 3,2$ months $-p=0,0024)$ and shorter OS $(20,6 \times 12,4$ months $-p=0,0017)$. The multivariate analysis found a numerical difference between the groups but statistical significance was not reached. Liu et al. [29] using this same method, the CellSearch System (the only one approved by FDA to analyze CTCs from metastatic breast, colon and prostate cancers) in a small study enrolling 78 women with newly diagnosed or recurrent ovarian epithelial cancer did not show a relationship between CTC counts and clinical outcomes. The absence of relation was explained considering the inabilities to enhance the phenotypic characteristics of CTCs. Pearl et al. (2014) refined the search considering what was named iCTCs (invasive CTCs). By using cell adhesion matrix 
(CAM) enrichment these researchers were able to raise sensitivity and specificity, obtaining positive results. There was a stronger relationship among OS, PFS and unfavorable clinical factors (tumor stage, debulking, platinum sensitivity) for iCTCs when compared to CA125 [28].

The controversial findings of these studies can be explained by the diversity of the enrolled population, sample size, different times of blood collection and diverse techniques used to isolate CTCs. Most these studies used only epithelial markers to identify CTCs, and is well established on literature that these cells sometimes lose their epithelial markers and express mesenchymal ones, as a manner to leave the primary tumor, invade the circulation and reach distant organs [29]. Here, we used a method that is marker independent and isolates CTCs by size. This method, called ISET (Isolation by Size of Epithelial Tumors; Rarecells $s^{\circ}$, France) allows to capture CTCs no matter in which phase of this evolution they are, if they express epithelial or mesenchymal markers. This explains why it was possible to follow-up the patient described here and to notice her tumor evolution by CTC analysis before imaging exams. It is important to notice that to our knowledge our group was the first to identify and characterize ovarian cancer CTCs using ISET. Besides availability for isolating CTCs, the method allows the researcher to identify tumor microemboli and to immunostain the cells for diverse surface markers, improving the possibility of further studies.

In summary, it is important to emphasize that in this case report the CTC analysis provided a very reliable prediction for recurrent disease, sometimes before the ascension of the CA125 levels, what may warrant CTCs as a complementary tool for OC follow-up. CTCs are not a perfect biomarker, but are of very important consideration, particularly in cases where CA125 cannot be trusted alone. The lack of statistical significance to the correlation between CA125 and CTC dosages demonstrates, to our point of view, that these tools could be evaluated together in the care of patients, especially the ones when follow-up can be troublesome.

However, it is necessary to address that any definite conclusions regarding the role of CTCs in Ovarian Cancer, specially by the usage of ISET as a novel modality for research, are not possible, and a prognostic evaluation of a series of cases is necessary to confirm our hypothesis. Thus, we do not intend to define CTCs as an individual biomarker for follow-up in ovarian cancer, but to determine them as a novel tool that could be very useful in cases where the current technology is not capable of defining alone the best care for patients.

\section{Conclusions}

As shown by recent studies, CTC counts is a valuable complementary tool in evaluation of recurrence in ovarian epithelial cancer, especially in when disease is not precisely measured by CA125, such as low-grade adenocarcinoma, very young patients, those with disease limited to the peritoneum and other patients that may present with a disparity between extent of disease and levels of CA125 [30, 31].

With the presented data, we would encourage studies to validate the use of CTCs counts in the clinical practice of ovarian epithelial cancer. For patients with low-grade adenocarcinoma, this indication should be carefully evaluated and may benefit decision-making.

\section{Acknowledgements}

The authors would like to thank Emne Abdallah and Alexcia Braun for the help with conduction of the analysis and readiness when necessary.

\section{Funding}

The evaluation of Circulating Tumor Cells for this project was funded by resources from FAPESP (Fundação de Amparo à Pesquisa do Estado de São Paulo). Diagnostic studies and treatments of the patient were self funded via a health insurance plan.

\section{Availability of data and materials}

Data sharing is not applicable to this article as no datasets were generated or analysed during the current study. All data regarding this case report is available at the medical records systems of our institution, including imaging studies, laboratorial results, written medical consultations, visits to the emergency service, visits to the chemotherapy facility and every contact that the patient had with our institution. Being a case report with a review of the literature, there is no available dataset on which the conclusions and inferences made by our group were built. The review of the literature was based on PubMed search for relevant data on the theme, and all references are listed in the body of the test. Upon request, the scientific papers cited in this text can be made available via Cloud Services.

\section{Author's contributions}

$M C$ and LC contributed equally to the writing of the manuscript. MC conducted the literature review and writing of the data supporting the study. LC conducted the final review of the manuscript and coordinated the experiments conducted. AG, SS, MF and AC contributed with patient care, review of the manuscript data regarding the case report and review of the literature. GB contributed directly with the care of this patient and review of patient specific data. VA and BR contributed with research specific

procedures, including specimen collection and processing. LC contributed with the final review and approval of the manuscript before submission. All authors read and approved the final manuscript.

\section{Competing interests}

The authors declare that they have no competing interests for the elaboration of this manuscript.

\section{Consent for publication}

The patient described in this case report gave written consent not only to voluntarily participate in the CTC evaluation program, but also for the publication of the data concerning her medical history and treatments at our institution. The consent for participation and usage of her data in our research is available at our institution.

Ethics approval and consent to participate

The evaluation of Circulating Tumor Cells was initiated under the protocol number 1367-10, by the approval of the Local Research Ethics Committee (CEP - Comitê de Ética e Pesquisa) at A.C.Camargo Cancer Hospital, São Paulo, Brazil.

\section{Publisher's Note}

Springer Nature remains neutral with regard to jurisdictional claims in published maps and institutional affiliations. 


\section{Author details}

Medical Oncology Department, A.C.Camargo Cancer Center, São Paulo, Brazil. ${ }^{2}$ International Research Center, A.C.Camargo Cancer Center, Rua Taguá, 440. Liberdade, São Paulo, SP 01508-010, Brazil. ${ }^{3}$ Gynecological Surgery Department, A.C.Camargo Cancer Center, São Paulo, Brazil.

Received: 12 January 2017 Accepted: 5 May 2017

Published online: 04 July 2017

\section{References}

1. Siegel R, Ma J, Zou Z, Jemal A. Cancer statistics, 2014. CA A Cancer J Clin. 2014;64(1):9-29.

2. Ledermann JA, Raja FA, Fotopoulou C, Gonzalez-Martin A, Colombo N, Sessa C, et al. Newly diagnosed and relapsed epithelial ovarian carcinoma: ESMO Clinical Practice Guidelines for diagnosis, treatment and follow-up. Ann Oncol. 2013;24 Suppl 6:vi24-32.

3. Chen WW, Ruiz B, Killeen JL, Coté TR, Wu XC, Correa CN, et al. Pathology and classification of ovarian tumors. Cancer. 2003:97(S10):2631-42.

4. Della Pepa C, Tonini G, Santini D, Losito S, Pisano C, Di Napoli M, et al. Low Grade Serous Ovarian Carcinoma: From the molecular characterization to the best therapeutic strategy. Cancer Treat Rev. 2014;41(2):136-43.

5. Romero I, Sun CC, Wong KK, Bast Jr RC, Gershenson DM. Low-grade serous carcinoma: New concepts and emerging therapies. Gynecol Oncol. 2013; 130(3):660-6.

6. Fader AN, Java J, Krivak TC, Bristow RE, Tergas Al, Bookman MA, et al. The prognostic significance of pre- and post-treatment CA-125 in grade 1 serous ovarian carcinoma: A Gynecologic Oncology Group study. Gynecol Oncol. 2014;132(3):560-5.

7. Markman M, Liu PY, Rothenberg ML, Monk BJ, Brady M, Alberts DS. Pretreatment CA-125 and Risk of Relapse in Advanced Ovarian Cancer. J Clin Oncol. 2006;24(9):1454-8.

8. Aghajanian C, Blank SV, Goff BA, Judson PL, Teneriello MG, Husain A, et al. OCEANS: A Randomized, Double-Blind, Placebo-Controlled Phase III Trial of Chemotherapy With or Without Bevacizumab in Patients With PlatinumSensitive Recurrent Epithelial Ovarian, Primary Peritoneal, or Fallopian Tube Cancer. J Clin Oncol. 2012;30(17):2039-45.

9. Hofman V, Bonnetaud C, Ilie MI, Vielh P, Vignaud JM, Fléjou JF, et al. Preoperative Circulating Tumor Cell Detection Using the Isolation by Size of Epithelial Tumor Cell Method for Patients with Lung Cancer Is a New Prognostic Biomarker. Clin Cancer Res. 2011;17(4):827-35.

10. Krebs MG, Hou J-M, Sloane R, Lancashire L, Priest L, Nonaka D, et al. Analysis of Circulating Tumor Cells in Patients with Non-small Cell Lung Cancer Using Epithelial Marker-Dependent and -Independent Approaches. J Thorac Oncol. 2012;7(2):306-15.

11. Chi DS, Venkatraman ES, Masson V, Hoskins WJ. The Ability of Preoperative Serum CA-125 to Predict Optimal Primary Tumor Cytoreduction in Stage III Epithelial Ovarian Carcinoma. Gynecol Oncol. 2000;77(2):227-31.

12. Tuxen MK, Sölétormos G, Dombernowsky P. Serum tumour marker CA 125 in monitoring of ovarian cancer during first-line chemotherapy. $\mathrm{Br} J$ Cancer. 2001;84(10):1301-7.

13. Gadducci A, Cosio S, Fanucchi A, Negri S, Cristofani R, Genazzani AR. The predictive and prognostic value of serum CA 125 half-life during paclitaxel/ platinum-based chemotherapy in patients with advanced ovarian carcinoma. Gynecol Oncol. 2004;93(1):131-6.

14. Zivanovic O, Sima CS, lasonos A, Bell-McGuinn KM, Sabbatini PJ, Leitao MM, et al. Exploratory analysis of serum CA-125 response to surgery and the risk of relapse in patients with FIGO stage IIIC ovarian cancer. Gynecol Oncol. 2009;115(2):209-14.

15. Tang A. Kondalsamy-Chennakesavan S, Ngan H, Zusterzeel P, Quinn M, Carter J, et al. Prognostic value of elevated preoperative serum CA125 in ovarian tumors of low malignant potential: A multinational collaborative study (ANZGOG0801). Gynecol Oncol. 2012;26(1):36-40.

16. Pelissier A, Bonneau C, Chéreau E, de La Motte Rouge T, Fourchotte V, Darai $E$, et al. CA125 kinetic parameters predict optimal cytoreduction in patients with advanced epithelial ovarian cancer treated with neoadjuvant chemotherapy. Gynecol Oncol. 2014;135(3):542-6.

17. Wilbaux M, Hénin E, Oza A, Colomban O, Pujade-Lauraine E, Freyer G, et al. Dynamic modeling in ovarian cancer: An original approach linking early changes in modeled longitudinal CA-125 kinetics and survival to help decisions in early drug development. Gynecol Oncol. 2014;133(3):460-6.
18. Johnson CC, Kessel B, Riley $T L$, Ragard LR, Williams CR, Xu J-L, et al. The epidemiology of CA-125 in women without evidence of ovarian cancer in the Prostate, Lung, Colorectal and Ovarian Cancer (PLCO) Screening Trial. Gynecol Oncol. 2008;110(3):383-9.

19. Terada KY, Elia J, Kim R, Carney M, Ahn HJ. Abnormal CA-125 levels in menopausal women without ovarian cancer. Gynecol Oncol. 2014;135(1):34-7.

20. Lee CK, Friedlander M, Brown C, Gebski VJ, Georgoulopoulos A, Vergote I, et al. Early Decline in Cancer Antigen 125 as a Surrogate for ProgressionFree Survival in Recurrent Ovarian Cancer. J Natl Cancer Inst. 2011;103(17): 1338-42.

21. Armstrong A, Otvos B, Singh S, Debernardo R. Evaluation of the cost of CA125 measurement, physical exam, and imaging in the diagnosis of recurrent ovarian cancer. Gynecol Oncol. 2013;131(3):503-7.

22. Fehm T, Becker S, Bachmann C, Beck V, Gebauer G, Banys M, et al. Detection of disseminated tumor cells in patients with gynecological cancers. Gynecol Oncol. 2006;103(3):942-7.

23. Liberko M, Kolostova K, Bobek V. Essentials of circulating tumor cells for clinical research and practice. Crit Rev Oncol Hematol. 2013;88(2):338-56.

24. Paterlini-Brechot $P$, Benali NL. Circulating tumor cells (CTC) detection: Clinical impact and future directions. Cancer Lett. 2006;253(2):180-204.

25. Braun S, Schindlbeck C, Hepp F, Janni W, Kentenich C, Riethmüller G, et al, Occult Tumor Cells in Bone Marrow of Patients With Locoregionally Restricted Ovarian Cancer Predict Early Distant Metastatic Relapse. J Clin Oncol. 2001:19(2):368-75

26. Riethdorf S, Wikman H, Pantel K. Review: Biological relevance of disseminated tumor cells in cancer patients. Int J Cancer. 2008;123(9):1991-2006.

27. Judson PL, Geller MA, Bliss RL, Boente MP, Downs Jr LS, Argenta PA, et al. Preoperative detection of peripherally circulating cancer cells and its prognostic significance in ovarian cancer. Gynecol Oncol. 2003;91(2):389-94.

28. Pearl ML, Zhao Q, Yang J, Dong H, Tulley S, Zhang Q, et al. Prognostic analysis of invasive circulating tumor cells (iCTCs) in epithelial ovarian cancer. Gynecol Oncol. 2014;134(3):581-90.

29. Paterlini-Bréchot P. Circulating Tumor Cells: Who is the Killer? Cancer Microenviron 2014;7(3):161-76

30. Herzog TJ, Vermorken JB, Pujade-Lauraine E, Provencher DM, JagielloGruszfeld A, Kong B, et al. Correlation between CA-125 serum level and response by RECIST in a phase III recurrent ovarian cancer study. Gynecol Oncol. 2011;122(2):350-5.

31. Romero-Laorden N, Olmos D, Fehm T, Garcia-Donas J, Diaz-Padilla I. Circulating and disseminated tumor cells in ovarian cancer: A systematic review. Gynecol Oncol. 2014;133(3):632-9.

\section{Submit your next manuscript to BioMed Central and we will help you at every step:}

- We accept pre-submission inquiries

- Our selector tool helps you to find the most relevant journal

- We provide round the clock customer support

- Convenient online submission

- Thorough peer review

- Inclusion in PubMed and all major indexing services

- Maximum visibility for your research

Submit your manuscript at www.biomedcentral.com/submit
) Biomed Central 